\title{
A new approach for self localization of wireless sensor network
}

\author{
O.P. Sahu ${ }^{1}$ and Tarun Dubey ${ }^{2}$ \\ ${ }^{1}$ Department of Electronics \& Communication Engineering, NIT, Kurukshetra (Haryana) India \\ ${ }^{2}$ Department of Electronics \& Communication Engineering, IIET, Bareilly (U.P.) India \\ tarundubey79@yahoo.co.in
}

\begin{abstract}
This paper presents a new approach for self localization of wireless sensors. Various algorithms have been proposed for localization of wireless sensor networks and are based on computational measurements. The suggested approaches estimate the location of sensors implemented with concepts of functionalities and future scopes. The proposed algorithm relies on a range measurement technique between a pair of nodes and their arrangement to cover maximum area surrounding the main node. Hence, a confident position of sensor is established. The evident advantage of the algorithm is that it requires less number of sensor nodes and saves the constructional cost of a wireless sensor network.
\end{abstract}

Keywords: localization, sensors, node, wireless sensor networks (WSN)

\section{Introduction}

Sensor networks are being used in large number of military and civil needs. Today WSN is a key technology for different types of environments and sensor networks are of particular importance when a large number of sensor nodes have to be placed in a given location. Localization is done when there is an uncertainty regarding location of some fixed or mobile devices. Localization is an area that has attracted much attention in the recent years. Location awareness is vital for wireless sensor networks since many applications like environmental monitoring, vehicle tracking and mapping depend on knowing the exact location of sensor nodes. Recently many localization techniques have been proposed to allow the nodes to estimate their own locations using information transmitted by set of sub nodes that know their positions. WSN play a major role towards sensing and computing within human supervision. Current researches in sensor networks have been primarily due to improvements in their performance and decrease in their cost (Messer \& Thehybrid Cramer Rao, 2006). As these sensors are inexpensive, tiny and untethered they can be deployed in large numbers. Some civilian applications of sensor networks include monitoring systems in vineyards, environmental habitats, community areas, traffic highways and smart bridges. Sensor networks are used for surveillance of armed troops their vehicles in battlefields as well as for detection, tracking and classification of enemy targets. Many sensor networking applications such as object tracking and supervision of devices measure signals that are functions of the geometry between the objects under surveillance and the sensor. Presently all the localization techniques suffer from one or the other problem related to accuracy, range, distribution and area. Other problems like energy efficiency and power consumption typically focus on minimizing the transmission energy only (Srinivasan \& Wu, 2007; Venkatesh, 2007; Wan et al., 2008), which is reasonable for long range applications where the transmission energy is a dominant factor in the total energy consumption spectrum. However in short range applications for wireless sensor networks, the total energy consumption is comparable and even dominates the transmission energy. The sensor network energy consumption includes the energy consumed by all the sensor nodes along with the signal path which also depends on distance and placement of nodes. Our aim is to develop a localization technique such that sensors determine their own positions after their placement. Such localization is typically achieved when each sensor can compute its range from neighbouring sensor further they can algorithmically be placed in a graph measured by these ranges in a desired coordinate system. This coordinate system is then used to perform location dependent tasks (Ceveher et al., 2007), such as target tracking and position estimation. Simulation is also done so that the required processing for performing localization over previous techniques is achieved for improvements thus the generated location information is used for wide range of industrial, environmental, civil and military applications.

\section{Localization}

The theory of localization justifies the concept as a mechanism to obtain location based information of a sensor in a coordinate system with respect to specific time, distance, received signal strength, time difference of arrival, angle of arrival, time of flight and lifetime related parametric values to locate the position of a particular object. For many applications like habitat monitoring, smart buildings, failure detection and target tracking, it is necessary to accurately orient the nodes with respect to a globally recognized coordinate system in order to record the data that is geographically useful and correct. Furthermore basic middle ware services such as routing and military target tracking generally rely on location related information. On one hand, the low cost of the nodes facilitates massive scale use and high parallel computations but on the other hand the performance of each node is power dependent and its reliability to
Research article (Rapid communication) Clndian Society for Education and Environment (iSee)
"Wireless sensor network" http://www.indjst.org
Sahu \& Dubey Indian J.Sci.Technol. 
communicate locally with neighbouring nodes. This specific application area makes it unrealistic to depend faithfully on exact placement and uniform arrangement (Venkatesh \& Michael Buehrer, 2007), of sensors. Therefore the use of globally accessible beacons or expensive GPS techniques to localize individual sensor is encouraged because the basic objective is to successfully self localize all the individual sensors in the world wide accepted coordinate system and different environments, as shown in Fig.1.

\section{Fig. 1. Localization using GPS technique}

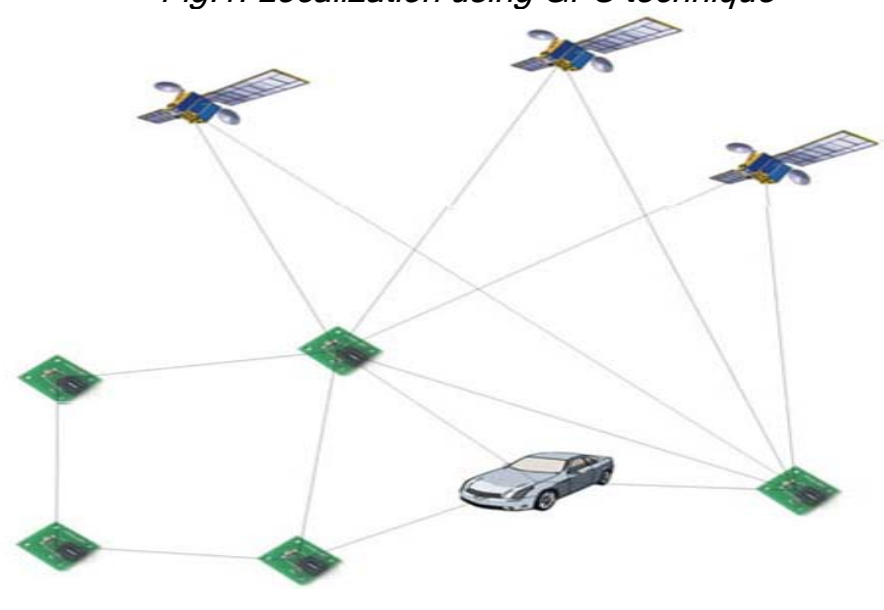

Wireless sensor network localization and its applications

Wireless sensor network being a significant technology is attracting considerable research (Biswas \& Ye, 2006), attention in recent years. It is one of the most important technologies of the 21st century. Recent advances in areas like wireless communication, electronics and MEMS (Adam Dunkels, 2007), have enabled the development of low cost, low power and multi functional sensor nodes that are small in size and communicate within short distances. These tiny sensor nodes (Joakim Eriksson et al., 2007), which consist of sensing elements, data processing chips and communicating components develop an idea for use of wireless sensor networks for real life. Sensor networks also show a significant improvement over traditional and conventional sensors. Cheap, smart and low cost sensors are networked through wireless links and can be deployed in large numbers to provide unprecedented opportunities for monitoring and controlling homes, cities, and the environment. Networked sensors have a broad spectrum of application in the defense area hence generating new capabilities for surveillance as well as other confidential tasks. Emerging applications (Jin et al., 2009), for wireless sensor networks also depend on automatic and accurate location of thousands of sensors. In environmental sensing applications such as bush fire surveillance, water quality monitoring, precision agriculture and indoor air quality monitoring it is clear that sensing data without knowing the location of a sensor is meaningless. However, location estimation may enable new areas such as inventory management, intrusion detection, traffic monitoring and telecare as well. In this work presented we have investigated the distribution of sensors based on a range estimation algorithm (Elnahrawy et al., 2009), for a wireless sensor network so that the distance of sub nodes is known prior to their placement.

\section{Restraints in localization algorithm design}

(a) Resource constraints: Sensor networks are typically quite resource starved. Nodes have rather weak processors, which make large computations difficult. Moreover, sensor nodes are battery powered; this means communication processing and sensing actions are all expensive as it actively reduces the lifespan of the node. Sensors have to be placed on a large scale along with hundreds or thousands of real working nodes in an environment. This fact has two important observations firstly the nodes must be cheap to fabricate with good power backup and secondly should be easy to deploy.

(b) Density of node: Node density is another important parameter to which localization algorithms are sensitive. Hop count based schemes generally require high node density so that the hop count approximation for distance becomes accurate. Similarly algorithms that depend on beacon nodes fail when the beacon density is low enough in a particular region. Thus while designing or analysing an algorithm it is quite important to note the algorithm's implicit density assumptions since high node density can sometimes be very expensive if not totally infeasible.

(c) Non convex topologies: In localization algorithms border nodes are a cause of concern (Chen et al., 2006), because less information is available through them and the information received is also of a lower quality. This problem can only be sorted, when a sensor network has a non convex shape which is always not desirable. Hence, shape of a sensor network plays a major role in collection of useful data.

(d) Terrain irregularities and environmental design: There are many environmental effects and irregularities that greatly affect the localization. Large rocks can block the line of sight or interfere with signals and introduce errors. Deployment on grass and sand can affect radio signals and acoustic range systems. While working indoors constructional features like walls can impede accurate measurements as well. All the above cited issues exist in real deployment of sensors therefore a sensor network should be able to cope with these limitations.
Research article (Rapid communication) CIndian Society for Education and Environment (iSee)
"Wireless sensor network" http://www.indjst.org
Sahu \& Dubey Indian J.Sci.Technol. 
(e) System architecture: This is the most important issue as it defines taxonomy (Mao Chen Liu et al., 2009; Sotiris Nikoletseas \& Paul G. Spirakis, 2009), for localization algorithms based on their results. Centralized algorithms are designed to run on a central machine with plenty of computational power. Sensor nodes gather environmental data and pass it back to a base station for analysis after which the computed positions are transported back into the sensor network. In contrast distributed algorithms (Jianfeng Qu et al., 2009), are designed to run in the network which uses parallel processing and inter node communication to compensate for the lack of centralized computing power. The distributed algorithms use a subset of the data to solve for each position independently, yielding an approximate result to locate a particular node.

\section{Proposed algorithm}

We have surveyed different systems in which the wireless nodes are mobile for the duration of their lifetime (Mao Chen Liu et al., 2009), having overall power dissipation. The area for the wireless mobile sensor network is less very often when compared with other networks like mobile networks, fixed node networks and wired networks A new algorithm is hereby suggested to cover more area. This algorithm will localize the sensors in a well defined manner to cover a particular geographic location with sensors covering a span of maximum area.

Step1- define nodes: $M, N 1$ and N2 Define range: $R$

\section{Step2 - compare distance among nodes}

\section{Step3 -if $N 1<R$, Place $N 1=R$ from $M$}

Step4- compare distance between $M$ and N2 If N2>R then compare distance between N1 and N2

\section{Step5 -if $N 2<R$ place $N 2$ from $N 1$ at range $R$}

(a) About the algorithm: We have chosen one main node which is connected to the system and two sub nodes. All are randomly placed in a particular area. This main node and pair of sub nodes is shown in Fig.2. Firstly we define node $\mathrm{M}$ which is the main node connected to system. N1 and N2 are randomly placed sub nodes. The sub nodes represent sensors and have sensing some range. In step one, distance between $\mathrm{M}$ and $\mathrm{N} 1$ is compared, if it is less than the defined range, $\mathrm{N} 1$ is placed at a distance $\mathrm{R}$ from $M$. In second step distance between $M$ and N2 is compared if it is greater than the defined range then the distance between $\mathrm{N} 1$ and $\mathrm{N} 2$ is compared, if this distance is less than the defined range $R$, we have to place the sub node at a distance R from N1.Using this approach we have tried to localize the sensors as shown in Fig. 3.

Fig.2. Randomly placed sensor nodes

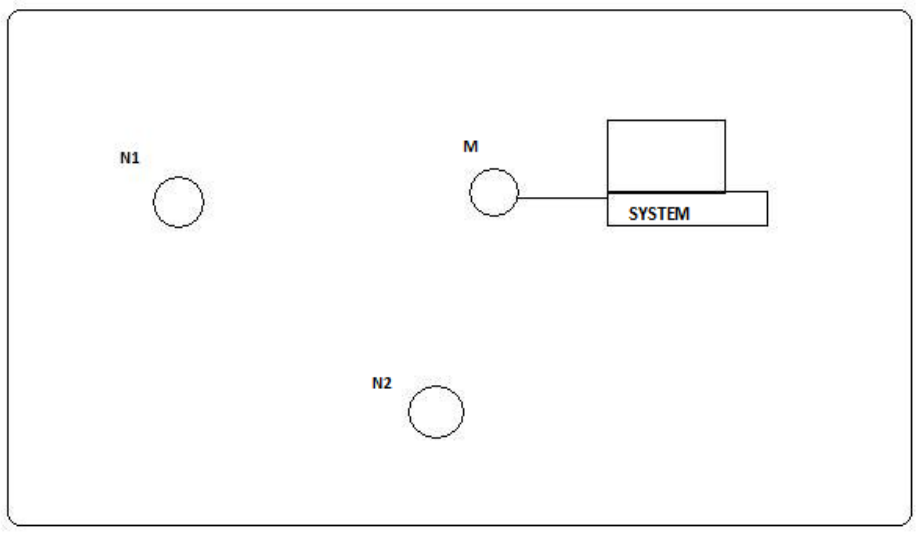

Fig.3. Placement of sensor nodes after applying algorithm for coverage of maximum area

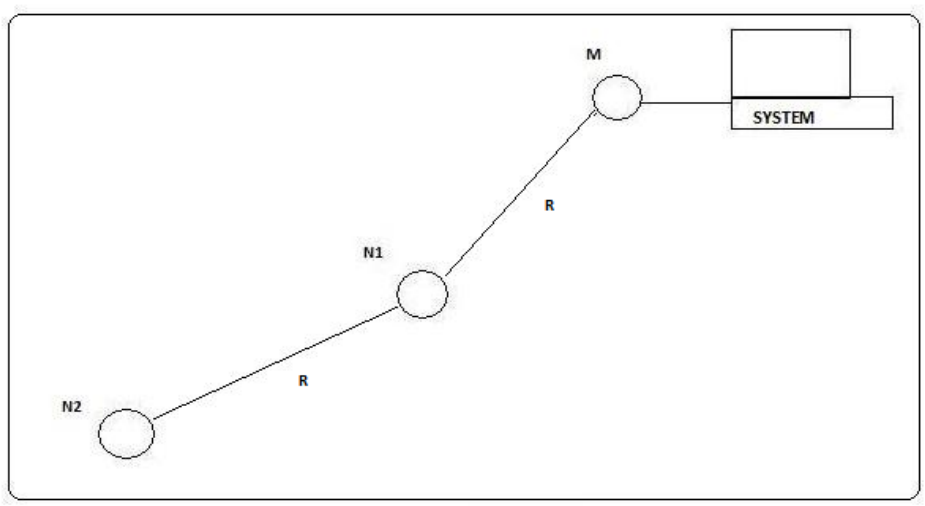

(b) Advantage of the algorithm: The advantage of this algorithm is vital since it requires lesser number of sensor nodes and still it can cover a larger area. This procedure is also cost effective as minimum number of sensors is used.

\section{Results and conclusions}

The localization algorithm is designed in $\mathrm{C}++$ language. Experimental results are shown in Fig.4. On applying the algorithm successful placement of paired sensor nodes $\mathrm{N} 1$ and N2 is achieved and maximum area is covered. The experimental value selected for sensor range $R$ is 50 meters and the results shown in the output exist within the specified range. The work highlighted in this paper is also useful for researchers working in this field as many issues still remain to be explored in the future, including how to design an optimal WSN and improve the location precision.
Research article (Rapid communication) CIndian Society for Education and Environment (iSee)
"Wireless sensor network" http://www.indjst.org
Sahu \& Dubey Indian J.Sci.Technol. 
Fig.4. Output of algorithm.

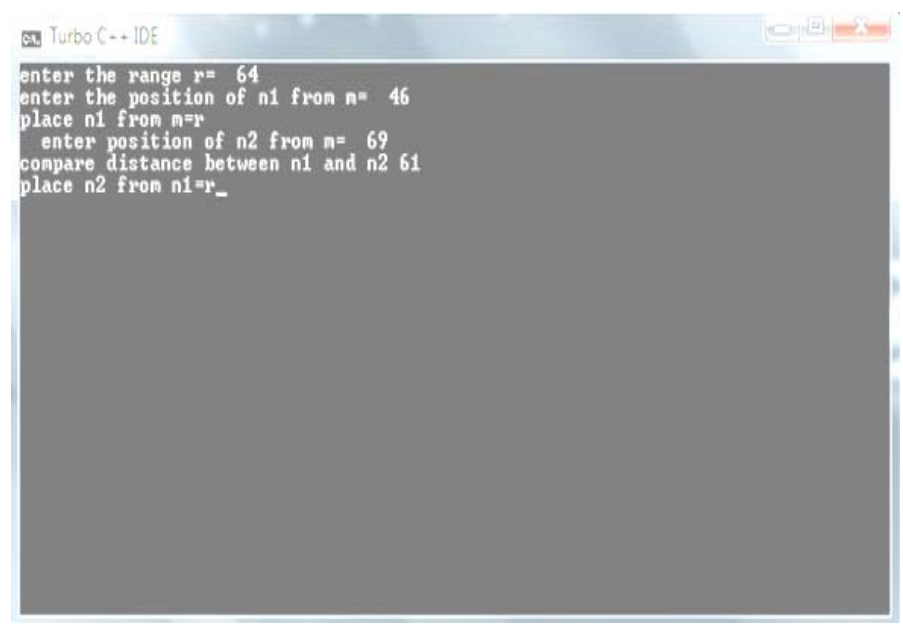

\section{References}

1. Adam Dunkels (2007) Lightweight layered communication stack for sensor networks. Proc. Eur. Conf. on Wireless Sensor Networks (EWSN), Netherlands. Vol. 91, No 8 pp. 1247- 1256.

2. Biswas $P$ and $Y e Y(2006) A$ distributed method for solving semi definite programs arising from adhoc wireless sensor network localization, in Multiscale Optimization Methods and applications J. Nonconvex Optim. Appl. Vol. 82, pp. 69-84.

3. Ceveher V, Chellapa R and McClellan J (2007) Gaussian approximations for energy-based detection and localization in sensor networks. IEEE Statistical Signal Processing Workshop. Vol.14, pp. 40

4. Chen Y, Francisco J, Trappe W and Martin RP (2006) A practical approach to landmark deployment for indoor localization. Proc. 3rd Annual IEEE Commun. Soc. Conf. on Sensor, Mesh \& Ad Hoc Commun. \& Networks (SECON).

5. Elnahrawy E, Li X and Martin RP (2009) The limits of localization using signal strength: a comparative study. Proc. Ist IEEE Intl. Conf. on Sensor \& Adhoc Commun. \& Networks (SECON).

6. Jianfeng Qu, Yi Chai and Simon X. Yang (2009) A real-time de-noising algorithm for e-noses in a wireless sensor network. J. Sensors. 9(2), 895-908.

7. Jin Z, Jian Ping, Yu Si, Wang Ya, Ping Z and Guang $L$ (2009) Survey on position based routing algorithms in wireless sensor networks. J. Algorithms. 2, 158182.

8. Joakim Eriksson, Adam Dunkels, Niclas Finne, Fredrik Österlind and Thiemo Voigt (2007) MSPSim an extensible simulator for msp430-equipped sensor boards. Proc. Eur. Conf. on Wireless Sensor Networks (EWSN), Poster/Demo session, Delft, Netherlands. Vol. 4373, pp. XIII, 358.

9. Mao Chen Liu, Ching Liang Dai, Chih Hua Chan and Chyan Chyi Wu (2009) Manufacture of a polyaniline

Research article (Rapid communication)

CIndian Society for Education and Environment (iSee)
"Wireless sensor network" http://www.indjst.org nanofiber ammonia sensor integrated with a readout Circuit using the CMOS-MEMS technique. J. Sensors. Vol.9 (2), pp. 869-880.

10. Messer $H$ and Thehybrid Cramer Rao (2006) lower bound Practice to theory. J. Sensor Array \& Multi Channel Processing. Vol.89, pp. 304-307.

11. Sotiris Nikoletseas and Paul G. Spirakis (2009) Review: probabilistic distributed algorithms for energy efficient routing and tracking. J. Wireless Sensor Networks Algorithms. Vol. 2(1), pp. 121-157.

12. Srinivasan A and Wu J (2007) A survey on secure localization in wireless sensor networks. Wireless and Mobile Communications, CRC Press/Taylor \& Francis Group, Boca Raton/London.

13. Venkatesh S (2007) The design and modelling of ultra-wideband position-location networks. Ph.D. Dissertation, Virginia Polytechnic Institute \& State Univ. (Virginia Tech).

14. Venkatesh S and Michael Buehrer R (2007) Multiple access insights from bounds on sensor localization. Mobile and Portable Radio Research Group (MPRG), Virginia Tech, Blacksburg.

15. Wan Z, Zhang J and Zhu H (2008) On energy-efficient and low latency media access control protocol for wireless sensor networks. Proc. IEEE WCNC. Vol.5, pp. 148-164.
Sahu \& Dubey Indian J.Sci.Technol. 